Pacific Journal of Mathematics

THE SPACE OF EXTENDED ORTHOMORPHISMS IN A RIESZ 


\title{
THE SPACE OF EXTENDED ORTHOMORPHISMS IN A RIESZ SPACE
}

\author{
B. De Pagter
}

\begin{abstract}
We study the space $\operatorname{Orth}^{\infty}(L)$ of extended orthomorphisms in an Archimedean Riesz space $L$ and its analogies with the complete ring of quotients of a commutative ring with unit element. It is shown that for any uniformly complete $f$-algebra $A$ with unit element, $\operatorname{Orth}^{\infty}(A)$ is isomorphic with the complete ring of quotients of $A$. Furthermore, it is proved that for any uniformly complete Riesz space $L$ the space $\operatorname{Orth}^{\infty}(L)$ is isomorphic to the lateral completion of $L$. Finally, it is shown that for any uniformly complete Riesz space $L$ the ring $\operatorname{Orth}^{\infty}(L)$ is von Neumann regular.
\end{abstract}

The main subject in this paper is the space $\operatorname{Orth}^{\infty}(L)$ of extended orthomorphisms in an Archimedean Riesz space $L$. By an extended orthomorphism we mean an order bounded linear mapping $\pi$ from an order dense ideal $D$ in $L$ into $L$ with the property that $\pi f \perp g$ for all $f \in D$ and $g \in L$ with $f \perp g$. As shown in [10], $\operatorname{Orth}^{\infty}(L)$ is an $\operatorname{Archimedean}$ $f$-algebra with unit element which is, in addition, laterally complete.

The definition of $\operatorname{Orth}^{\infty}(L)$ for an Archimedean Riesz space is in some sense analogous to the definition of the complete ring of quotients $Q(R)$ of a commutative ring $R$ with unit element (see [8], §2.3). A natural thing to do, therefore, is to compare these two objects for Archimedean $f$-algebras with unit element. In $\$ 2$ of this paper it is proved that for any uniformly complete $f$-algebra $A$ with unit element, the algebras $\operatorname{Orth}^{\infty}(A)$ and $Q(A)$ are indeed isomorphic.

For any $f$-algebra $A=C(X)$, where $X$ is a completely regular Hausdorff space, the complete ring of quotients of $A$ is precisely the lateral completion $A^{\lambda}$ of $A$. So, by the above-mentioned result, in this case $\operatorname{Orth}^{\infty}(A)$ is the lateral completion of $A$. In $\S 3$ we study the relation between $\operatorname{Orth}^{\infty}(L)$ and the lateral completion $L^{\lambda}$ for an arbitrary Archimedean Riesz space, and it will be shown that $\operatorname{Orth}^{\infty}(L)=L^{\lambda}$ holds for uniformly complete Riesz spaces.

Another interesting property of the ring of quotients $Q(R)$ of a semiprime commutative ring $R$ with unit element is that $Q(R)$ is von Neumann regular. In the last section of this paper it will be shown that $\operatorname{Orth}^{\infty}(L)$ is a von Neumann regular $f$-algebra for any uniformly complete Riesz space $L$. 
1. Preliminaries. For terminology concerning the general theory of Riesz spaces and order bounded operators we refer to [1] and [11]. We assume that all Riesz spaces considered are Archimedean. The order bounded linear mapping $\pi$ from an order dense ideal $D$ in the Archimedean Riesz space $L$ into $L$ is called an extended orthomorphism whenever $\pi f \perp g$ for all $f \in D$ and $g \in L$ with $f \perp g$ (see [10], §1 or [9], Chapter IV, $\S 1)$. The collection of all extended orthomorphisms in $L$ will be denoted by $\operatorname{Orth}^{\infty}(L)$. Since any extended orthomorphism is order continuous ([10], Theorem 1.3), it is natural to consider two extended orthomorphisms as equal if they agree on their common domain of definition or, equivalently, if they agree on some order dense ideal in $L$. With respect to the pointwise operations and ordering, $\operatorname{Orth}^{\infty}(L)$ is an Archimedean Riesz space.

An element $\pi \in \operatorname{Orth}^{\infty}(L)$ which is defined on the whole space $L$ is called an orthomorphism in $L$. The collection of all orthomorphisms in $L$ is denoted by $\operatorname{Orth}(L)$, and $\operatorname{Orth}(L)$ is a Riesz subspace of $\operatorname{Orth}^{\infty}(L)$. Properties of $\operatorname{Orth}(L)$ can be found in [9] and [12].

A Riesz space $A$ is called a Riesz algebra (or a lattice ordered algebra) if there exists in $A$ an associative multiplication such that $A$ is an algebra with the additional property that $u v \geq 0$ for all $0 \leq u, v \in A$. The Riesz algebra $A$ is called an f-algebra if $u \wedge v=0$ in $A$ implies that $(w u) \wedge v=$ $(u w) \wedge v=0$ for all $0 \leq w \in A$. For the general theory of $f$-algebras we refer the reader to [4], [7], [9] and [12]. In particular we recall that any Archimedean $f$-algebra is commutative. Observe that for any $f \in A$ the mapping $\pi_{f}$, which assigns to each $g \in A$ the element $f g$, is an orthomorphism in $A$. If $A$ has a unit element, then any orthomorphism in $A$ is of the form $\pi_{f}$ for some $f \in A$.

Both Riesz spaces $\operatorname{Orth}^{\infty}(L)$ and $\operatorname{Orth}(L)$ can be given an $f$-algebra structure by taking composition as multiplication (see [10], §1). Clearly $\operatorname{Orth}(L)$ is an $f$-subalgebra of $\operatorname{Orth}^{\infty}(L)$. Observe that the identity mapping in $L$ serves as unit element in $\operatorname{Orth}^{\infty}(L)$ as well as in $\operatorname{Orth}(L)$.

The Riesz space $L$ is called laterally complete if every positive disjoint system in $L$ has a supremum in $L$ (e.g. [1], Chapter 7). It is proved in [10], Theorem 1.4, that $\operatorname{Orth}^{\infty}(L)$ is laterally complete. Any Archimedean laterally complete Riesz space has the projection property (see [1], Theorem 23.4). The Riesz space $L$ is called universally complete if $L$ is laterally complete and Dedekind complete. Every Archimedean Riesz space $L$ has a universal completion $L^{u}$, i.e., there exists a universally complete Riesz space $L^{u}$ such that $L$ can be identified with an order dense Riesz subspace of $L^{u}$ (see [11], Theorem 50.8). In fact $L^{u}$ is a Riesz space $C^{\infty}(\Omega)$ for some appropriate extremally disconnected compact Hausdorff space $\Omega$. This 
shows in particular that for any weak order unit $e$ in $L^{u}$ there exists an $f$-algebra multiplication in $L^{u}$ with $e$ as unit element.

The laterally complete Riesz space $L^{\lambda}$ is called a lateral completion of $L$ if $L$ can be identified with an order dense Riesz subspace of $L^{\lambda}$. The lateral completion, as well as the universal completion, is essentially unique (this follows e.g. from [1], Theorem 23.16), and therefore $L^{\lambda}$ can be considered as the intersection of all laterally complete Riesz subspaces of $L^{u}$ in which $L$ is contained.

For the sake of convenience we recall that the Riesz subspace $K$ of $L$ is called order dense in $L$ whenever for any $0<f \in L$ there exists $g \in K$ such that $0<g \leq f$ or, equivalently, for any $0 \leq f \in L$ we have $f=$ $\sup \{g \in K: 0 \leq g \leq f\}$. Clearly, if $K$ is order dense in $L$ and $f_{\tau} \downarrow 0$ in $K$ then $f_{\tau} \downarrow 0$ in $L$ as well. Furthermore, if $K$ is order dense in $L$, then every band in $L$ is equal to the band generated in $L$ by some band in $K$, and any band in $K$ is the intersection of $K$ with some band in $L$ (see [4], Théorème 11.1.15).

The next proposition provides a useful characterization of the lateral completion. Recall that the linear mapping $P$ from $L$ into itself is called an order projection whenever $P^{2}=P$ and $0 \leq P u \leq u$ for all $0 \leq u \in L$.

Proposition 1.1. Let $L$ be an Archimedean Riesz space with universal completion $L^{u}$ and lateral completion $L^{\lambda} \subset L^{u}$. For any $0 \leq f \in L^{u}$ the following two conditions are equivalent.

(i) $f \in L^{\lambda}$.

(ii) There exist disjoint order projections $P_{\alpha}$ in $L^{u}$ and $0 \leq u_{\alpha} \in L$ such that $f=\sup _{\alpha} P_{\alpha} u_{\alpha}$.

Proof. The above proposition can be deduced from [2], §5 and [3], Theorem 1. However, it can also be proved directly as follows. Define $M^{+}$ to be the subset of $L^{u}$ consisting of all $0 \leq f \in L^{u}$ for which there exist disjoint order projections $P_{\alpha}$ in $L^{u}$ and $0 \leq u_{\alpha} \in L$ such that $f=\sup _{\alpha} P_{\alpha} u_{\alpha}$. Let $M$ be the set of all $f$ - $g$ with $f, g \in M^{+}$. Now it is easily checked that $M$ is a laterally complete Riesz subspace of $L^{u}$ and $L \subset M \subset L^{u}$. Assume now that $K$ is a laterally complete Riesz subspace of $L^{u}$ such that $L \subset K \subset L^{u}$. We assert that $M \subset K$. Indeed, as noted before, since $K$ is laterally complete, $K$ has the projection property. Furthermore, since $K$ is order dense in $L^{u}$, the order projections in $K$ are precisely the restrictions of the order projections in $L^{u}$. Hence, if $P$ is an order projection in $L^{u}$ and $0 \leq u \in L$, then $P u \in K$. Now take $0 \leq f \in M$. Then by the definition of $M, f=\sup _{\alpha} P_{\alpha} u_{\alpha}$, where $\left\{P_{\alpha}\right\}$ are disjoint order projections in $L^{u}$ and $0 \leq u_{\alpha} \in L$. As observed, $P_{\alpha} u_{\alpha} \in K$ for all $\alpha$, and therefore the lateral 
completeness of $K$ implies $f \in K$. Hence $M \subset K$, and we may conclude that $M$ is the lateral completion of $L$.

We proceed with some further terminology. Given $v \geq 0$ in the Riesz space $L$, the sequence $\left\{f_{n}: n=1,2, \ldots\right\}$ in $L$ is called $v$-uniformly convergent to $f \in L$ if for every real number $\varepsilon>0$ there exists a natural number $n_{\varepsilon}$ such that $\left|f-f_{n}\right| \leq \varepsilon v$ for all $n \geq N_{\varepsilon}$. This will be denoted by $f_{n} \rightarrow f$ ( $v$-uniformly). If $f_{n} \rightarrow f$ ( $v$-uniformly) for some $0 \leq v \in L$, then the sequence $\left\{f_{n}\right\}$ is called (relatively) uniformly convergent to $f$, which is denoted by $f_{n} \rightarrow f$ (r.u.). The notion of ( $v$-) uniform Cauchy sequence is defined in the obvious way. The Riesz space $L$ is called uniformly complete if every uniform Cauchy sequence in $L$ has a unique limit. For details we refer to [11], $\$ \S 16$ and 63.

Finally we recall some facts about commutative rings. For the proofs we refer to [8], §2.3. Let $R$ be a commutative ring with unit element. The ring ideal $I$ in $R$ is called dense if it follows from $s \in R$ and $s r=0$ for all $r \in I$ that $s=0$. A mapping $q$ from a dense ring ideal $I \subset R$ into $R$ with the property that $q(s r)=s q(r)$ for all $s \in R$ and $r \in I$ is called a fraction in $R$. Two fractions will be considered equal if they agree on their common domain of definition. Note that two fractions are equal iff they agree on some dense ring ideal in $R$. The collection of all fractions in $R$, with the above identification, is denoted by $Q(R)$. With respect to pointwise addition and composition as multiplication it turns out that $Q(R)$ is a commutative ring with unit element (see [8], §2.3, Proposition $1)$. Now $Q(R)$ is called the complete ring of quotients of $R$. By assigning to each $r \in R$ the element $q_{r} \in Q(R)$ defined by $q_{r}(s)=r s$ for all $s \in R, R$ is embedded in $Q(R)$ as a subring. Note that $R$ consists precisely of those $q \in Q(R)$ which have as domain the whole ring $R$. If $q \in Q(R)$ with domain $I$ and $r \in I$, then $q \cdot q_{r}$ is the multiplication by the element $q(r)$ in $R$. Indeed, let the dense ideal $J$ in $R$ be defined by $J=\{s \in R$ : $r s \in I\}$. For any $s \in J$ we have $\left(q \cdot q_{r}\right)(s)=q(r s)=q(r) s$, and hence $q \cdot q_{r}$ agrees with multiplication by $r$ on the dense ideal $J$.

If the ring $R$ is semiprime (i.e., $r^{n}=0$ implies $r=0$ ), then $Q(R)$ is a von Neumann regular ring, i.e., for any $r \in R$ there exists an element $r_{1} \in R$ such that $r=r_{1} r^{2}$ (see [8], §2.4, Proposition 1).

2. $\operatorname{Orth}^{\infty}(A)$ as the complete ring of quotients of $A$. Let $A$ be an Archimedean $f$-algebra with unit element. As before, for any $f \in A$ we denote by $\pi_{f}$ the orthomorphism in $A$ defined by $\pi_{f} g=f g$ for all $g \in A$. Since $A$ has a unit element, any orthomorphism in $A$ is of the form $\pi_{f}$ for some $f \in A$, i.e., $A$ and $\operatorname{Orth}(A)$ can be identified as $f$-algebras. Since 
$\operatorname{Orth}(A)$ is an $f$-subalgebra of $\operatorname{Orth}^{\infty}(A)$, we may consider $A$ as an $f$-subalgebra of $\operatorname{Orth}^{\infty}(A)$.

Suppose $\pi \in \operatorname{Orth}^{\infty}(A)$ with domain $D$ and take $f \in D$. Then the domain of $\pi \pi_{f}$ is $D_{1}=\{g \in A: f g \in D\}$, and for any $g \in D_{1}$ we have

$$
\begin{aligned}
\left(\pi \pi_{f}\right)(g) & =\pi(f g)=\pi\left(\pi_{g} f\right)=\left(\pi \pi_{g}\right)(f)=\left(\pi_{g} \pi\right)(f) \\
& =\pi_{g}(\pi f)=(\pi f) g .
\end{aligned}
$$

Hence the extended orthomorphism $\pi \pi_{f}$ agrees on the order dense ideal $D_{1}$ in $A$ with the multiplication by $\pi f$, i.e., with the orthomorphism $\pi_{\pi f}$. We conclude that $\pi \pi_{f}=\pi_{\pi f}$ and, hence, if we identify $f$ and $\pi_{f}$ (i.e., we consider $A$ as an $f$-subalgebra of $\left.\operatorname{Orth}^{\infty}(A)\right)$, then $\pi f=\pi \cdot f$. In other words, $\pi f$ may be considered as the image of $f$ under $\pi$ as well as the product of $\pi$ and $f$ in $\operatorname{Orth}^{\infty}(A)$.

Since $A$ is a commutative ring with unit element, $A$ is also a subring of the complete ring of quotients $Q(A)$. Note that $Q(A)$ is in fact an algebra and $A$ is a subalgebra of $Q(A)$. Now we shall study the relation between $Q(A)$ and $\operatorname{Orth}^{\infty}(A)$. We start with a definition.

DeFinition 2.1. The algebra homomorphism $\Phi$ from $\operatorname{Orth}^{\infty}(A)$ into $Q(A)$ is called natural if $\Phi$ leaves $A$ invariant (i.e., if $\Phi\left(\pi_{f}\right)=q_{f}$ for all $f \in A)$.

The next theorem shows us the existence of a natural homomorphism.

THEOREM 2.2. If $A$ is an Archimedean f-algebra with unit element, then there exists a unique natural homomorphism $\Phi$ from $\operatorname{Orth}^{\infty}(A)$ into $Q(A)$. Moreover, $\Phi$ is injective.

Proof. We first define a natural homomorphism. Let $\pi \in \operatorname{Orth}^{\infty}(A)$ be given with domain $D$. Denote by $(D)$ the ring ideal generated by $D$, i.e.,

$$
(D)=\left\{\sum_{l=1}^{n} r_{i} d_{l}: r_{i} \in A, d_{l} \in D, i=1, \ldots, n, n \in \mathbf{N}\right\} .
$$

We define the mapping $q$ from $(D)$ into $A$ by $q\left(\sum_{t=1}^{n} r_{i} d_{i}\right)=\sum_{l=1}^{n} r_{i} \pi d_{i}$. In order to see what $q$ is well defined, suppose $\sum_{l=1}^{n} r_{l} d_{l}=0\left(r_{l} \in A, d_{l} \in D\right)$. In $\operatorname{Orth}^{\infty}(A)$ we then have $\sum_{i=1}^{n} \pi_{r_{i}} \pi_{d_{l}}=0$, so $\pi\left(\sum_{i=1}^{n} \pi_{r_{i}} \pi_{d_{1}}\right)=0$, which implies $\sum_{i=1}^{n} \pi_{r_{i}} \pi \pi_{d_{t}}=0$. Since $d_{l} \in D$, it follows from the remarks above that $\pi \pi_{d_{l}}=\pi_{\pi d_{l}}$ and therefore $\sum_{i=1}^{n} \pi_{r_{t}} \pi_{\pi d_{l}}=0$, i.e., $\sum_{i=1}^{n} r_{l} \pi d_{l}=0$ in $A$. Hence $q$ is well defined. It is clear that $q$ is an $A$-linear mapping from ( $D$ ) into $A$, and it follows from $D \subset(D)$ that $(D)$ is a dense ring ideal in $A$. Therefore $q \in Q(A)$. We now show that $q$ is independent of the domain 
of definition $D$ of $\pi$. For this purpose, suppose $\pi^{\prime} \in \operatorname{Orth}^{\infty}(A)$ with domain $D^{\prime}$ and $\pi=\pi^{\prime}$ on $D \cap D^{\prime}$. Let the mapping $q^{\prime}$ from $\left(D^{\prime}\right)$ into $A$ be defined as above. It is clear from the definition that $q=q^{\prime}$ on $\left(D \cap D^{\prime}\right)$, and since $\left(D \cap D^{\prime}\right)$ is a dense ring ideal, this implies $q=q^{\prime}$ in $Q(A)$. Defining $\Phi(\pi)=q$, it is evident that $\Phi$ is an injective linear mapping from $\operatorname{Orth}^{\infty}(A)$ into $Q(A)$ which leaves $A$ invariant. We shall show now that $\Phi$ is a homomorphism. Take $\pi_{1}, \pi_{2} \in \operatorname{Orth}^{\infty}(A)$ with domains $D_{1}$ and $D_{2}$, respectively. Then the domain of $\pi=\pi_{1} \pi_{2}$ is $\pi_{2}^{-1}\left(D_{1}\right)$ $=\left\{f \in D_{2}: \pi_{2} f \in D_{1}\right\}$. Put $q_{1}=\Phi\left(\pi_{1}\right), q_{2}=\Phi\left(\pi_{2}\right)$ and $q=\Phi(\pi)$. The domain of $q_{1} q_{2}$ is $q_{2}^{-1}\left(\left(D_{1}\right)\right)$, and since $\left(\pi_{2}^{-1}\left(D_{1}\right)\right) \subset q_{2}^{-1}\left(\left(D_{1}\right)\right)$, it is sufficient to prove that $q=q_{1} q_{2}$ on $\left(\pi_{2}^{-1}\left(D_{1}\right)\right)$. This, however, follows immediately from the definition of $\Phi$. We may conclude, therefore, that $\Phi$ is a natural homomorphism.

Finally, we show $\Phi$ is unique. To this end suppose $\Psi$ is a natural homomorphism from $\operatorname{Orth}^{\infty}(A)$ into $Q(A)$, and take $\pi \in \operatorname{Orth}^{\infty}(A)$ with domain $D$. Put $q=\Psi(\pi)$ and let $I$ be the domain of $q$. Take $r \in I \cdot(D)$, i.e., $r=\sum_{l=1}^{n} r_{i} d_{i}$ with $d_{i} \in D$ and $r_{i} \in I(i=1,2, \ldots, n)$. Then

$$
\begin{aligned}
q(r) & =\sum_{i=1}^{n} d_{i} q\left(r_{i}\right)=\sum_{i=1}^{n}\left(q_{d_{i}} q\right)\left(r_{i}\right)=\sum_{i=1}^{n}\left\{\Psi\left(\pi_{d_{i}} \pi\right)\right\}\left(r_{i}\right) \\
& =\sum_{i=1}^{n}\left\{\Psi\left(\pi_{\pi d_{i}}\right)\right\}\left(r_{i}\right)=\sum_{i=1}^{n} q_{\pi d_{i}}\left(r_{i}\right) \\
& =\sum_{i=1}^{n} r_{i} \pi d_{i}=\{\Phi(\pi)\}(r) .
\end{aligned}
$$

This shows $q=\Phi(\pi)$ on the dense ring ideal $I \cdot(D)$, so $q=\Phi(\pi)$ in $Q(A)$. Hence $\Psi=\Phi$, and the theorem is proved completely.

The natural homomorphism $\Phi$ is in general not surjective. This is shown in the next example.

EXAMPLE 2.3. Let $A$ be the collection of all realvalued continuous functions on $[0,1]$ which are piecewise polynomials (finitely many pieces). With respect to the pointwise operations, $A$ is an Archimedean $f$-algebra with unit element. Let $\pi \in \operatorname{Orth}^{\infty}(A)$ be defined by $(\pi f)(x)=$ $(x+1) f(x)$ for all $f \in A$ and $0 \leq x \leq 1$. Now $\pi \in A$ implies $\Phi(\pi)=q$ is also multiplication by $x+1$. In $Q(A)$ the element $q$ has an inverse (multiplication by the function $(x+1)^{-1}$ on the dense ring ideal $\left.(x+1) A\right)$. However, $\pi$ does not have an inverse in $\operatorname{Orth}^{\infty}(A)$. Indeed, suppose $\pi_{1}$ is the inverse of $\pi$ in $\operatorname{Orth}^{\infty}(A)$ with domain $D_{1}$. Then $\pi\left(\pi_{1} f\right)=f$ for all $f \in D_{1}$. For any $0<u \in D_{1}$ we have $(x+1)\left(\pi_{1} u\right)(x)=u(x)$ for all 
$x \in[0,1]$, which implies $0 \leq \pi_{1} u \leq u$, so $\pi_{1} u \in D_{1}$. Therefore $(x+1)$. $\left(\pi_{1}^{2} u\right)(x)=\left(\pi_{1} u\right)(x)$, from which we deduce that $u(x)=(x+1)^{2}$. $\left(\pi_{1} u\right)^{2}(x)$ on $[0,1]$. Repeating this argument we conclude that there exists a sequence $u_{n} \in A(n=1,2, \ldots)$ such that $u(x)=(x+1)^{n} u_{n}(x)$ for all $0 \leq x \leq 1$ and all $n$, which is impossible. Hence the natural homomorphism cannot be surjective.

If $A$ is a uniformly complete $f$-algebra, then the situation improves. This is shown in the following theorem.

THEOREM 2.4. For any uniformly complete f-algebra $A$ with unit element, the natural homomorphism $\Phi$ is an isomorphism from $\operatorname{Orth}^{\infty}(A)$ onto $Q(A)$.

Proof. We need only show that $\Phi$ is surjective. Take $q \in Q(A)$ with domain $I$ and define the ideal $D$ in $A$ to be the collection of all $f \in A$ for which there exist $g_{1}, \ldots, g_{n} \in I$ such that $|f| \leq \sum_{i=1}^{n} g_{i}^{2}$. We assert that $D \subset I$. Indeed, if $|f| \leq \sum_{i=1}^{n} g_{i}^{2}\left(g_{i} \in I\right)$ then $0 \leq f^{+} \leq \sum_{t=1}^{n} g_{i}^{2}$, so, by the Riesz decomposition property ([11], Corollary 15.6 (ii)), $f^{+}=\sum_{i=1}^{n} u_{t}$ with $0 \leq u_{i} \leq g_{i}^{2}(i=1, \ldots, n)$. Since $A$ is uniformly complete and has a unit element it follows from [7], Corollary 3.12 (ii), that there exist $h_{i} \in A$ $(i=1, \ldots, n)$ such that $u_{i}=h_{i} g_{i}$. Hence $u_{i} \in I(i=1, \ldots, n)$, which implies $f^{+} \in I$. Similarly we find that $f^{-} \in I$ and therefore $f \in I$.

Now we show that $D$ is order dense in $A$. Suppose $g \in A$ is such that $g \perp f$ for all $f \in D$. Then $g \perp h^{2}$ for all $h \in I$ so $g h^{2}=0$ for all $h \in I$. This implies $g^{2} h^{2}=0$ and, hence, $g h=0$ for all $h \in I$ (recall that any Archimedean $f$-algebra with unit element is semiprime; see e.g. [7], Proposition 3.2 (iii)). Since $I$ is a dense ring ideal we see that $g=0$.

Let $\pi$ denote the restriction of $q$ to the order dense ideal $D$. If $f \in D$ and $g \in A$ such that $f \perp g$, then $f g=0$ and so $q(f) g=q(f g)=0$. This implies $q(f) \perp g$, i.e., $\pi f \perp g$ (again using that $A$ is semiprime). Hence $\pi \in \operatorname{Orth}^{\infty}(A)$.

We claim that $\Phi(\pi)=q$. Indeed, let $(D)$ be the ring ideal generated by $D$ in $A$ and take $r \in(D)$, i.e., $r=\sum_{i=1}^{n} r_{i} d_{i}$ with $r_{i} \in A$ and $d_{i} \in D$ $(i=1, \ldots, n)$. Then

$$
\Phi(\pi)(r)=\sum_{i=1}^{n} r_{i}\left(\pi d_{l}\right)=\sum_{i=1}^{n} r_{i}\left(q d_{i}\right)=q\left(\sum_{i=1}^{n} r_{l} d_{i}\right)=q(r) .
$$

Hence $\Phi(\pi)=q$ on $(D)$, and therefore $\Phi(\pi)=q$ in $Q(A)$. We have thus proved that $\Phi$ is surjective, which concludes the proof of the theorem. 
It follows immediately from the above theorem that in this situation the complete ring of quotients $Q(A)$ can be equipped with a partial ordering in such a way that $Q(A)$ becomes an $f$-algebra. In fact we define $q_{1} \leq q_{2}$ whenever $\Phi^{-1}\left(q_{1}\right) \leq \Phi^{-1}\left(q_{2}\right)$ holds in $\operatorname{Orth}^{\infty}(A)$. It is easy to see that $q_{1} \leq q_{2}$ in $Q(A)$ is then equivalent to $q_{1}(u) \leq q_{2}(u)$ for all positive elements $u$ in their common domain of definition. This remark yields the following result.

Corollary 2.5. Let $A$ be a uniformly complete f-algebra with unit element. For $q_{1}, q_{2} \in Q(A)$ we define $q_{1} \leq q_{2}$ whenever $q_{1}(u) \leq q_{2}(u)$ holds for all positive elements in the common domain of definition. Then $Q(A)$ is an Archimedean f-algebra with respect to this ordering.

As noted in $\S 1$, the complete ring of quotients $Q(R)$ of a commutative semiprime ring $R$ with unit element, is von Neumann regular. It follows, therefore, from Theorem 2.4 that for any uniformly complete $f$-algebra $A$ with unit element the $f$-algebra $\operatorname{Orth}^{\infty}(A)$ is von Neumann regular. In $\S 4$ we shall show that $\operatorname{Orth}^{\infty}(L)$ is in fact von Neumann regular for any uniformly complete Riesz space $L$.

For any completely regular Hausdorff space $X$ we denote by $\mathcal{G}(X)$ the collection of all dense open subsets of $X$. The complete ring of quotients of the $f$-algebra $C(X)$ is the space $C[\mathcal{G}(X)]$ of all continuous functions defined on some $G$ in $\mathcal{G}(X)$ (with identification of functions which agree on some member of $\mathcal{G}(X)$ ). It is not difficult to see that $C[\mathcal{G}(X)]$ is also equal to the lateral completion of $C(X)$ (for a survey of all kinds of completions of the space $C(X)$ see [6]). Hence for the Riesz space $C(X)$ the lateral completion is precisely the space $\operatorname{Orth}^{\infty}(C(X))$. In the next section we shall study the relation between $\operatorname{Orth}^{\infty}(L)$ and $L^{\lambda}$ for arbitrary Riesz spaces $L$.

3. $\operatorname{Orth}^{\infty}(L)$ as the lateral completion of $L$. Let $L$ be an Archimedean Riesz space with universal completion $L^{u}$. We choose in $L$ some maximal disjoint system $\left\{e_{\tau}\right\}$, which will be fixed in all of the following considerations. Then $e=\sup e_{\tau}$ exists in $L^{u}$ and $e$ is a weak order unit in $L^{u}$. We consider in $L^{u}$ the $f$-algebra multiplication for which $e$ is the unit element. As usual for $f$-algebras with unit element, we may identify $\operatorname{Orth}\left(L^{u}\right)$ and $L^{u}$.

Take $0 \leq \pi \in \operatorname{Orth}^{\infty}(L)$ with domain $D \subset L$. Since $D$ is order dense in $L$, the universal completion of $D$ is $L^{u}$. Since $\pi$ is order continuous, $\pi$ 
can be extended to an order continuous Riesz homomorphism $\pi^{\wedge}$ from the ideal $D^{\wedge}$ generated by $D$ in $L^{u}$ into $L^{u}$ (observe that $D^{\wedge}$ is the Dedekind completion of $D$ ). Now it follows for [1], Theorem 23.16, that $\pi^{\wedge}$ can be extended to an order continuous Riesz homomorphism $\pi^{*}$ from $L^{u}$ into itself, and it is easy to see that $\pi^{*} \in \operatorname{Orth}\left(L^{u}\right)$. Putting $\pi^{*}=\Psi(\pi)$, we define an injective Riesz homomorphism from $\operatorname{Orth}^{\infty}(L)$ into $\operatorname{Orth}\left(L^{u}\right)$. Using the above-mentioned identification of $L^{u}$ and $\operatorname{Orth}\left(L^{u}\right)$, we thus get an embedding of $\operatorname{Orth}^{\infty}(L)$ into $L^{u}$. In fact, $\operatorname{Orth}^{\infty}(L)$ corresponds to the set of all $p \in L^{u}$ for which there exists an order dense ideal $D$ in $L$ such that $p f \in L$ for all $f \in D$.

As before we denote by $L^{\lambda}$ the lateral completion of $L$. Then both $L^{\lambda}$ and $\operatorname{Orth}^{\infty}(L)$ are laterally complete Riesz subspaces of $L^{u}$. We shall now show that $\operatorname{Orth}^{\infty}(L) \subset L^{\lambda}$. The following observation will be used in the proof of the next theorem. Let $A$ be an Archimedean $f$-algebra with unit element $e$ and suppose $P$ is an order projection in $A$. If we put $p=P e$, then we have $P f=p f$ for all $f \in A$.

THEOREM 3.1. For any Archimedean Riesz space $L$ we have $\operatorname{Orth}^{\infty}(L)$ $\subset L^{\lambda} \subset L^{u}$.

Proof. In this proof we use the multiplication in $L^{u}$ introduced above, and we identify $\operatorname{Orth}^{\infty}(L)$ with an $f$-subalgebra of $L^{u}$. Let $0<p \in$ $\operatorname{Orth}^{\infty}(L)$ be given with domain $D$, i.e., $p f \in L$ for all $f \in D$. Given the band $B \neq\{0\}$ in $L^{u}$, there exists $0<u \in B \cap D$ such that $0<u \leq e_{\tau}$ for some $e_{\tau}$. Let $n$ be a natural number with the property that $\left(n u-e_{\tau}\right)^{+}>0$ and put $B_{1}=\left\{\left(n u-e_{\tau}\right)^{+}\right\}^{d d}$ in $L^{u}$. Denote by $P_{1}$ the order projection in $L^{u}$ onto $B_{1}$ and put $p_{1}=P_{1} e$. Note that it follows from $p_{1} e=p_{1}$ and $p_{1} \in\left\{e_{\tau}\right\}^{d d}$ that $p_{1} e_{\tau}=p_{1}$. Since $n u \wedge e_{\tau} \in D$, the element $w=p\left(n u \wedge e_{\tau}\right)$ satisfies $w \in L$. Now

$$
\begin{aligned}
P_{1} w & =p_{1} p\left(n u \wedge e_{\tau}\right)=p p_{1}\left\{e_{\tau}-\left(n u-e_{\tau}\right)^{-}\right\} \\
& =p p_{1}=P_{1} p .
\end{aligned}
$$

We thus have proved that for any band $B \neq\{0\}$ in $L^{u}$ there exist a band $B_{1}$ in $L^{u}$ and an element $0 \leq w \in L$ such that $\{0\} \neq B_{1} \subset B$ and $P_{1} w=$ $P_{1} p$, where $P_{1}$ denotes the order projection in $L^{u}$ onto $B_{1}$. Now it follows by a standard argument that condition (ii) of Proposition 1.1 is satisfied, and we may conclude, therefore, that $p \in L^{\lambda}$.

The inclusion $\operatorname{Orth}^{\infty}(L) \subset L^{\lambda}$ can be proper, as shown by the following example. 
EXAMPLE 3.2. Let $L$ be the Riesz space of all piecewise linear real continuous functions on $[0,1]$. Since $L$ is order dense in $C([0,1])$, the universal completion $L^{u}$ of $L$ is the same as the universal completion of $C([0,1])$. Therefore $L^{u}$ is the space of all real continuous functions which are defined on some dense open subset of $[0,1]$ (with identification of functions which agree on some dense open subset). It is not difficult to see that $L^{\lambda}$ consists of all real functions $f$ defined on some open dense subset $S$ of $[0,1]$ which are locally linear, i.e., for any $x \in S$ there exists an open interval around $x$ on which $f$ is linear. However, $\operatorname{Orth}^{\infty}(L)$ consists of those functions in $L^{\lambda}$ which are locally constant.

RemarK 3.3. Let $A$ be an Archimedean $f$-algebra with unit element $e$. Since $e$ is a weak unit in $A$, there exists an $f$-algebra multiplication in $A^{u}$ for which $e$ is the unit element. In other words, the multiplication in $A$ can be extended to an $f$-algebra multiplication in $A^{u}$. As above, we consider $\operatorname{Orth}^{\infty}(A)$ as an $f$-subalgebra of $A^{u}$. Now the natural embedding of $A$ into $\operatorname{Orth}^{\infty}(A)$ is precisely the inclusion of $A$ in $A^{u}$, and so in this situation we have $A \subset \operatorname{Orth}^{\infty}(A) \subset A^{\lambda}$. Since $\operatorname{Orth}^{\infty}(A)$ is laterally complete, this implies $\operatorname{Orth}^{\infty}(A)=A^{\lambda}$.

We shall show now that $\operatorname{Orth}^{\infty}(L)=L^{\lambda}$ holds for any uniformly complete Riesz space $L$. To this end we need the following lemmas.

LEMMA 3.4. Let $M$ be a Riesz space with the projection property, $L$ a Riesz subspace of $M$ and $I$ an ideal in L. Take $0 \leq e \in I$ and $0 \leq u \in L$, and let $P_{e}$ be the order projection in $M$ onto $\{e\}^{d d}$. Then there exist mutually disjoint components $\left\{\boldsymbol{v}_{n}: n=0,1, \ldots\right\}$ of elements of $I$ in bands generated by elements of $I$ such that $\sup v_{n}=P_{e} u$.

Proof. For $n=0,1, \ldots$ we define the bands $B_{n}=\left\{(n e-u)^{+}\right\}^{d d}$. Note that $(n e-u)^{+} \in I$ for all $n$. Since $B_{n}=\left\{(e-u / n)^{+}\right\}^{d d}(n \neq 0)$, it follows from $(e-u / n)^{+} \uparrow e$ that $B_{n} \uparrow\{e\}^{d d}$ and, therefore, $P_{n} \uparrow P_{e}$, where $P_{n}$ denotes the order projection onto $B_{n}$. Observe that $B_{0}=\{0\}$, so $P_{0}=0$. Now define $v_{n}=\left(P_{n+1}-P_{n}\right) u$, i.e., $v_{n}$ is the component of $u$ in the band $B_{n+1} \cap B_{n}^{d}(n=0,1, \ldots)$. Clearly, $\left\{v_{n}: n=0,1, \ldots\right\}$ is a disjoint sequence in $M$, and for $N=1,2, \ldots$ we have

$$
\sup _{0 \leq n \leq N} v_{n}=\sum_{n=0}^{N}\left(P_{n+1}-P_{n}\right) u=P_{N+1} u-P_{0} u=P_{N+1} u \uparrow P_{e} u .
$$


Hence $\sup v_{n}=u$. If we now define $u_{n}=u \wedge n e$ for all $n=0,1, \ldots$, then $v_{n}=\left(P_{n+1}-P_{n}\right) u_{n+1}$. Indeed,

$$
\begin{aligned}
\left(P_{n+1}-P_{n}\right) u_{n+1} & =\left(P_{n+1}-P_{n}\right)\left\{u-(u-(n+1) e)^{+}\right\} \\
& =\left(P_{n+1}-P_{n}\right) u-\left(P_{n+1}-P_{n}\right)(u-(n+1) e)^{+} \\
& =\left(P_{n+1}-P_{n}\right) u=v_{n} .
\end{aligned}
$$

Therefore each $v_{n}$ is a component of the element $u_{n+1} \in I$.

Lemma 3.5. Let the Archimedean Riesz space L be an order dense Riesz subspace of the laterally complete Archimedean Riesz space $M$. Let I be an order dense ideal in $L$, and suppose $K$ is a laterally complete Riesz subspace of $M$ such that $I \subset K$. Then $L \subset K$.

Proof. Since $M$ and $K$ are laterally complete, both $M$ and $K$ have the projection property. Let $\left\{e_{\tau}\right\}$ be a maximal disjoint system in $I$. Then $\left\{e_{\tau}\right\}$ is also a maximal disjoint system in $M$. If we denote the order projection in $M$ onto $\left\{e_{\tau}\right\}^{d d}$ by $P_{\tau}$, then we have for any $0 \leq u \in L$ that $u=\sup P_{\tau} u$. Since $K$ is laterally complete, it is sufficient to show that $P_{\tau} u \in K$ for all $\tau$. For any fixed $\tau$ there exists, by Lemma 3.4 , a disjoint sequence $\left\{v_{n}\right.$ : $n=0,1, \ldots\}$ in $M$ consisting of components of elements of $I$ in bands generated by elements of $I$ such that $P_{\tau} u=\sup v_{n}$. Since $K$ has the projection property and $K$ is order dense in $M$, we have $v_{n} \in K$ for all $n$. Now it follows from the lateral completeness of $K$ that $P_{\tau} u \in K$. This concludes the proof of the lemma.

As above, let $\left\{e_{\tau}\right\}$ be some fixed maximal disjoint system in the Archimedean Riesz space $L$, and consider in $L^{u}$ the $f$-algebra multiplication with $e=\sup e_{\tau}$ as unit element. We identify $\operatorname{Orth}^{\infty}(L)$ with an $f$-subalgebra of $L^{u}$. It follows from Theorem 3.1 that we then have the following situation:

$L$

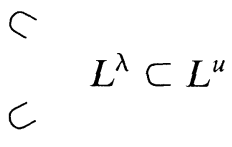

$\operatorname{Orth}^{\infty}(L)$

THEOREM 3.6. If $L$ is a uniformly complete Riesz space, then $L \subset$ $\operatorname{Orth}^{\infty}(L)$, and therefore $\operatorname{Orth}^{\infty}(L)=L^{\lambda}$. 
Proof. Denote by $I_{\tau}$ the principal ideal in $L$ generated by $e_{\tau}$ and put $I=\bigoplus_{\tau} I_{\tau}$. Clearly $I$ is an order dense ideal in $L$. It follows from Lemma 3.5 that it is sufficient to show that $I \subset \operatorname{Orth}^{\infty}(L)$; therefore if we show $I_{\tau} \subset \operatorname{Orth}^{\infty}(L)$ for each $\tau$, we are done. To this end fix $\tau$ and take $0 \leq f \in I_{\tau}$. Let $\pi_{f} \in \operatorname{Orth}^{\infty}\left(L^{u}\right)$ be the multiplication by $f$ in $L^{u}$. Since $I_{\tau}$ is uniformly complete and $e_{\tau}$ is a strong order unit in $I_{\tau}$, there exists $\pi \in \operatorname{Orth}\left(I_{\tau}\right)$ such that $\pi e_{\tau}=f$ (this follows immediately from the Yosida representation of $I_{\tau}$ as some $C(X)$ space; see [11], Theorem 45.4. The existence of the orthomorphism $\pi$ can also be proved without using representation theory; see [12], Remark 19.5 (ii)). We now extend $\pi$ to the order dense ideal $I_{\tau} \oplus I_{\tau}^{d}$ by defining $\pi=0$ on $I_{\tau}^{d}$. It is clear now that $\pi \in \operatorname{Orth}^{\infty}(L)$, and we denote the extension of $\pi$ to $L^{u}$ by $\pi$ again. It follows from the definition of $\pi$ that $\pi e_{\mu}=\pi_{f} e_{\mu}$ for all $\mu$, so we may conclude $\pi_{f}=\pi \in \operatorname{Orth}^{\infty}(L)$.

The following two results are immediate corollaries of the above theorem.

COROLlaRY 3.7. If $L$ is a uniformly complete Riesz space, then there exists a multiplication in $L^{\lambda}$ such that $L^{\lambda}$ is an Archimedean f-algebra with unit element.

COROllary 3.8. Let $L$ be an order dense Riesz subspace of the uniformly complete Riesz space $M$. Then any $\pi \in \operatorname{Orth}^{\infty}(L)$ has an extension $\pi^{*} \in \operatorname{Orth}^{\infty}(M)$.

It follows in particular from Theorem 3.6 that $\operatorname{Orth}^{\infty}(L)=\operatorname{Orth}\left(L^{\lambda}\right)$ holds for any uniformly complete Riesz space $L$. If we do not assume that $L$ is uniformly complete, then we can still prove one inclusion.

Theorem 3.9. For any Archimedean Riesz space $L$ we have $\operatorname{Orth}^{\infty}(L)$ $\subset \operatorname{Orth}\left(L^{\lambda}\right)$. In other words, any $\pi \in \operatorname{Orth}^{\infty}(L)$ can be extended to an orthomorphism in $L^{\lambda}$.

Proof. Suppose $0 \leq p \in \operatorname{Orth}^{\infty}(L)$ with domain $D$, i.e., $D$ is an order dense ideal in $L$ and $p f \in L$ for all $f \in D$. We have to show $p f \in L^{\lambda}$ for all $f \in L^{\lambda}$. To this end take $0 \leq f \in L^{\lambda}$. By Proposition 1.1 there exist disjoint order projections $P_{\alpha}$ in $L^{u}$ and elements $0 \leq u_{\alpha} \in L$ such that $f=\sup P_{\alpha} u_{\alpha}$. A moment's reflection shows we may assume each $P_{\alpha}$ is the order projection onto the band in $L^{u}$ generated by an element $0 \leq v_{\alpha} \in D$. Since $p f=\sup p P_{\alpha} u_{\alpha}$, it is sufficient to show $p P_{\alpha} u_{\alpha} \in L^{\lambda}$ for each $\alpha$. For 
this purpose fix $\alpha$ and denote by $Q_{n}$ the order projection in $L^{u}$ onto the band generated by $\left(n v_{\alpha}-u_{\alpha}\right)^{+}(n=0,1, \ldots)$. Clearly $Q_{n} \uparrow P_{\alpha}$. Now define $w_{n}=\left(Q_{n+1}-Q_{n}\right) u_{\alpha}(n=0,1, \ldots)$. Then $\left\{w_{n}: n=0,1, \ldots\right\}$ is a disjoint sequence and $\sup w_{n}=P_{\alpha} u_{\alpha}$, which implies $p P_{\alpha} u_{\alpha}=\sup p w_{n}$. Therefore it is sufficient to show that $p w_{n} \in L^{\lambda}$ for all $n$. Observe now that

$$
w_{n}=\left(Q_{n+1}-Q_{n}\right) u_{\alpha}=\left(Q_{n+1}-Q_{n}\right)\left\{u_{\alpha} \wedge(n+1) v_{\alpha}\right\},
$$

so $p w_{n}=\left(Q_{n+1}-Q_{n}\right) p\left\{u_{\alpha} \wedge(n+1) v_{\alpha}\right\}$. It follows from $v_{\alpha} \in D$ that $p\left\{u_{\alpha} \wedge(n+1) v_{\alpha}\right\} \in L$, and since $L^{\lambda}$ has the projection property, we conclude that $p w_{n} \in L^{\lambda}$.

The following corollary is immediate.

COROLlary 3.10. If $L$ is an Archimedean laterally complete Riesz space then $\operatorname{Orth}^{\infty}(L)=\operatorname{Orth}(L)$.

Corollary 3.11. For any Archimedean Riesz space $L$ we have that $\operatorname{Orth}^{\infty}\left(\operatorname{Orth}^{\infty}(L)\right)=\operatorname{Orth}^{\infty}(L)$.

Proof. Since $\operatorname{Orth}^{\infty}(L)$ is laterally complete, it follows from the above that $\operatorname{Orth}^{\infty}\left(\operatorname{Orth}^{\infty}(L)\right)=\operatorname{Orth}\left(\operatorname{Orth}^{\infty}(L)\right)$. Furthermore, since $\operatorname{Orth}^{\infty}(L)$ is an Archimedean $f$-algebra with unit element it follows that $\operatorname{Orth}\left(\operatorname{Orth}^{\infty}(L)\right)=\operatorname{Orth}^{\infty}(L)$ (usual identification).

REMARK 3.11. It follows in particular from Theorem 3.6 that any uniformly complete Riesz space $L$ can be embedded as a Riesz subspace in $\operatorname{Orth}^{\infty}(L)$. Recently this result has also been proved by M. Duhoux and M. Meyer [5] (Theorem 2.14 and Corollary 2.15). One of the main differences between their approach and ours is that we always consider $\operatorname{Orth}^{\infty}(L)$ as an $f$-subalgebra of $L^{u}$, whereas they do not make use of the multiplicative structure of $L^{u}$. In the same paper also the results of Corollaries 3.10 and 3.11 appear (see [5], Remarks 2.8.2 and 2.13.2).

4. Some algebraic properties of $\operatorname{Orth}^{\infty}(L)$. As we saw in $\S 2$, if $A$ is a uniformly complete $f$-algebra with unit element, then $\operatorname{Orth}^{\infty}(A)$ is algebra isomorphic to the complete ring of quotients $Q(A)$. Since $A$ is semiprime, $Q(A)$ is von Neumann regular (see [8], §2.4, Proposition 1), therefore $\operatorname{Orth}^{\infty}(A)$ is von Neumann regular in this case. It is natural to ask whether $\operatorname{Orth}^{\infty}(L)$ is von Neumann regular for each Archimedean Riesz space $L$. In general the answer is negative, as shown by Example 
2.3. The main purpose of this section is to prove that for any uniformly complete Riesz space $L$ the $f$-algebra $\operatorname{Orth}^{\infty}(L)$ is von Neumann regular.

First note that $\operatorname{Orth}^{\infty}(L)$ is in general not uniformly complete, not even if $L$ itself is uniformly complete. Since $\operatorname{Orth}^{\infty}(L)$ has the projection property, uniform completeness of $\operatorname{Orth}^{\infty}(L)$ implies Dedekind completeness (see [11], Theorem 42.6), so we then have $\operatorname{Orth}^{\infty}(L)=L^{u}$. It is shown in [10], Theorem 1.4 (i), that if $L$ is Dedekind complete, then $\operatorname{Orth}^{\infty}(L)$ is likewise Dedekind complete. Although $\operatorname{Orth}^{\infty}(L)$ is not uniformly complete in general, it does have a 'local' completeness property.

Proposition 4.1. Let $L$ be a uniformly complete Riesz space and suppose $\left\{\pi_{n}: n=1,2, \ldots\right\}$ is a uniform Cauchy sequence in $\operatorname{Orth}^{\infty}(L)$ such that all $\pi_{n}$ have a common dense domain $D$. Then there exists an element $\pi$ in $\operatorname{Orth}^{\infty}(L)$ such that $\pi_{n} \rightarrow \pi$ (r.u.) in $\operatorname{Orth}^{\infty}(L)$.

Proof. The proof is straightforward. Suppose $\left\{\pi_{n}\right\}$ is a $\pi_{0}$-uniform Cauchy sequence for some $0 \leq \pi_{0} \in \operatorname{Orth}^{\infty}(L)$ with domain $D_{0}$. Then $\left|\pi_{n}-\pi_{m}\right| \leq \varepsilon_{n} \pi_{0}$ for all $m \geq n$ and some sequence of real numbers $\varepsilon_{n} \downarrow 0$. Hence for any $f \in D \cap D_{0}$ and $m \geq n$ we have $\left|\pi_{n} f-\pi_{m} f\right| \leq \varepsilon_{n} \pi_{0}|f|$, which shows $\left\{\pi_{n} f\right\}$ is a uniform Cauchy sequence in $L$. Since $L$ is uniformly complete $\left\{\pi_{n} f\right\}$ has a uniform limit $\pi f$ in $L$. In this way we define a mapping $\pi$ from $D \cap D_{0}$ into $L$, and it is easy to see that $\pi \in \operatorname{Orth}^{\infty}(L)$ and $\pi_{n} \rightarrow \pi$ ( $\pi_{0}$-uniformly).

As is well known, if $A$ is a uniformly complete if-algebra with unit element $e$, then any element $u$ in $A$, such that $u \geq e$, has an inverse in $A$, and for any $0 \leq u \in A$ the square root, $\sqrt{u}$, exists in $A$ (see [7], Theorems 3.4 and 3.9, or [12], Theorems 11.1 and 11.5). We shall now prove similar results for $\operatorname{Orth}^{\infty}(L)$.

THEOREM 4.2. For any uniformly complete Riesz space $L$ the following statements hold.

(i) If $\pi \in \operatorname{Orth}^{\infty}(L)$ such that $\pi \geq I$, then $\pi^{-1}$ exists in $\operatorname{Orth}^{\infty}(L)$.

(ii) If $0 \leq \pi \in \operatorname{Orth}^{\infty}(L)$, then $\sqrt{\pi}$ exists in $\operatorname{Orth}^{\infty}(L)$.

Proof. (i) The proof goes along the same lines as the proof of the corresponding statement in uniformly complete $f$-algebras with unit element. Suppose first that $I \leq \pi \leq \alpha I$ for some $\alpha>1$. For $N=1,2, \ldots$ define

$$
\sigma_{N}=\sum_{n=0}^{N^{\prime}}\left(I-\alpha^{-1} \pi\right)^{n}
$$


If we denote the domain of $\pi$ by $D$, then the domain of $\sigma_{N}$ is likewise $D$. Furthermore $\left\{\sigma_{N}: N=1,2, \ldots\right\}$ is an $I$-uniform Cauchy sequence in $\operatorname{Orth}^{\infty}(L)$, so by the above proposition there exists $\sigma \in \operatorname{Orth}^{\infty}(L)$ such that $\sigma_{N} \rightarrow \sigma$ ( $I$-uniformly). Now it is clear that $\pi^{-1}=\alpha^{-1} \sigma$. Observe that the domain of $\sigma$ is again $D$. Now assume $\pi \in \operatorname{Orth}^{\infty}(L)$ with domain $D$ is such that $\pi \geq I$, and define $\pi_{n}=\pi \wedge n I(n=1,2, \ldots)$. By the above, $\pi_{n}^{-1}$ exists for all $n=1,2, \ldots$, and the domain of each $\pi_{n}^{-1}$ is $D$. Since $\left\{\pi_{n}^{-1}\right\}$ is an $I$-uniform Cauchy sequence, it follows again from the above proposition that there exists $\pi_{0} \in \operatorname{Orth}^{\infty}(L)$ such that $\pi_{n}^{-1} \rightarrow \pi_{0}$ (I-uniformly). Clearly $\pi_{0}=\pi^{-1}$. Note that the domain of $\pi^{-1}$ is again $D$.

(ii) Similar.

In order to prove that $\operatorname{Orth}^{\infty}(L)$ is von Neumann regular, we need a lemma. First note the following. Suppose $\pi \in \operatorname{Orth}^{\infty}(L)$ with domain $D_{0}$. Then by the domain of $\pi^{2}$ we shall mean the order dense ideal $\pi^{-1}\left(D_{0}\right)=$ $\left\{f \in D_{0}: \pi f \in D_{0}\right\}$, although it may happen that $\pi^{2}$ can be extended to an order dense ideal which is larger than $\pi^{-1}\left(D_{0}\right)$. We shall use the same terminology when dealing with higher powers of $\pi$.

LEMMA 4.3. Let $L$ be a uniformly complete Riesz space and suppose $0 \leq \pi \in \operatorname{Orth}^{\infty}(L)$ with domain $D_{0}$. If we denote the domain of $\pi^{4}$ by $D$, then the order ideal $J=\left\{f \in L:|f| \leq \pi^{2} u\right.$ for some $\left.u \in D\right\}$ is contained in $\pi\left(D_{0}\right)$.

Proof. It is sufficient to show that $0 \leq v \leq \pi^{2} u$ with $0 \leq u \in D$ implies there exists $w \in D_{0}$ such that $\pi w=v$. For $n=1,2, \ldots$ it follows from $\pi+I / n \geq I / n$ and from the above theorem that $(\pi+I / n)^{-1}$ exists in $\operatorname{Orth}^{\infty}(L)$ and the domain of $(\pi+I / n)^{-1}$ is again $D_{0}$. Putting $w_{n}=$ $(\pi+I / n)^{-1} v(n=1,2, \ldots)$, it follows from $(\pi+I / n)^{-1} \leq n I$ that $w_{n} \in D$ for all $n$. For $m \geq n$ we then have

$$
\begin{aligned}
0 & \leq w_{m}-w_{n}=\left(\pi+\frac{1}{m} I\right)^{-1} v-\left(\pi+\frac{1}{n} I\right)^{-1} v \\
& =\left(\pi+\frac{1}{m} I\right)^{-1}\left(\pi+\frac{1}{n} I\right)^{-1}\left(\frac{1}{n}-\frac{1}{m}\right) v \\
& \leq\left(\frac{1}{n}-\frac{1}{m}\right)\left(\pi+\frac{1}{m} I\right)^{-1}\left(\pi+\frac{1}{n} I\right)^{-1} \pi^{2} u \\
& \leq\left(\frac{1}{n}-\frac{1}{m}\right)\left(\pi+\frac{1}{m} I\right)^{-1}\left(\pi+\frac{1}{n} I\right)^{-1}\left(\pi+\frac{1}{n} I\right)\left(\pi+\frac{1}{m} I\right) u \\
& =\left(\frac{1}{n}-\frac{1}{m}\right) u .
\end{aligned}
$$


Hence $\left\{w_{n}: n=1,2, \ldots\right\}$ is a $u$-uniform Cauchy sequence in $L$. Since $L$ is uniformly complete, there exists $w \in L$ such that $w_{n} \rightarrow w$ (u-uniformly). Note that it follows from $0 \leq u \in D$ and $0 \leq w_{n} \in D(n=1,2, \ldots)$ that $w \in D$ and, hence, $w \in D_{0}$. We assert that $\pi w=v$. Indeed, since $\pi+I / n$ $\rightarrow \pi$ ( $I$-uniformly) and $w_{n} \rightarrow w$ (u-uniformly), we have $(\pi+I / n) w_{n} \rightarrow \pi w$ (r.u.). On the other hand,

$$
(\pi+I / n) w_{n}=(\pi+I / n)(\pi+I / n)^{-1} v=v
$$

for all $n=1,2, \ldots$. Hence $\pi w=v$.

THEOREM 4.5. For any uniformly complete Riesz space $L$ the f-algebra $\operatorname{Orth}^{\infty}(L)$ is von Neumann regular (i.e., for any $\pi \in \operatorname{Orth}^{\infty}(L)$ there exists $\pi_{1} \in \operatorname{Orth}^{\infty}(L)$ such that $\left.\pi=\pi_{1} \pi^{2}\right)$.

Proof. Take $0 \leq \pi \in \operatorname{Orth}^{\infty}(L)$ with domain $D_{0}$ and let $D$ be the domain of $\pi^{4}$. By the above lemma, the ideal $J=\left\{f \in L:|f| \leq \pi^{2} u\right.$ for some $0 \leq u \in D\}$ satisfies $J \subset \pi\left(D_{0}\right)$. Let $N_{\pi}$ be the kernel of $\pi$, i.e., $N_{\pi}=\left\{f \in D_{0}: \pi f=0\right\}$, and define the ideal $D_{1}$ by $D_{1}=\pi^{-1}(J)$. If we now put $E=\pi\left(D_{1} \cap N_{\pi}^{d}\right)$, then $E$ is an ideal in $L$. Indeed, suppose $0 \leq v \leq \pi u$ for some $0 \leq u \in D_{1} \cap N_{\pi}^{d}$. Then $\pi u \in J$ so $v \in J$, which implies $v=\pi w$ for some $0 \leq w \in D_{0}$ with $0 \leq w \leq u$. We have thus found an element $0 \leq w \in D_{1} \cap N_{\pi}^{d}$ such that $v=\pi w$, therefore $v \in E$.

Now observe that the restriction of $\pi$ to $D_{1} \cap N_{\pi}^{d}$ is an injective mapping from $D_{1} \cap N_{\pi}^{d}$ onto $E$. We can define, therefore, the mapping $\pi_{1}$ from $E \oplus E^{d}$ into $L$ as follows. If $g \in E$, then $g=\pi f$ for a unique $f \in D_{1} \cap N_{\pi}^{d}$, and we put $\pi_{1} g=f$. For any $g \in E^{d}$ we define $\pi_{1} g=0$. The mapping $\pi_{1}$ is clearly linear and we will now show that $\pi_{1} \in \operatorname{Orth}^{\infty}(L)$. Note already that the domain $E \oplus E^{d}$ is order dense in $L$. Now assume $g \in E \oplus E^{d}$ and $h \in L$ are such that $g \perp h$. Then $g=g_{1}+g_{2}$ for some $g_{1} \in E$ and $g_{2} \in E^{d}$. Clearly $g_{1} \perp h$ and $g_{1}=\pi f_{1}$ for some $f_{1} \in D_{1} \cap N_{\pi}^{d}$. It follows from $\pi\left(\left|f_{1}\right| \wedge|h|\right) \leq\left|g_{1}\right|$ that $\left(\left|f_{1}\right| \wedge|h|\right) \perp h$, therefore $\left|f_{1}\right| \wedge$ $|h| \in N_{\pi}$. On the other hand, $f_{1} \in D_{1} \cap N_{\pi}^{d}$ implies $\left|f_{1}\right| \wedge|h| \in N_{\pi}^{d}$, therefore $\left|f_{1}\right| \wedge|h|=0$, i.e., $\pi_{1} g_{1} \perp h$. This shows $\pi_{1} g \perp h$, and we conclude that $\pi_{1} \in \operatorname{Orth}^{\infty}(L)$.

We claim $\pi=\pi_{1} \pi^{2}$ in $\operatorname{Orth}^{\infty}(L)$. It follows immediately from the definition of $\pi_{1}$ that $\pi f=\pi \pi_{1} \pi f$ holds for all $f \in N_{\pi} \oplus\left(D_{1} \cap N_{\pi}^{d}\right)=$ $D_{1} \cap\left(N_{\pi} \oplus N_{\pi}^{d}\right)$. Hence, if we can show $D_{1}$ is order dense in $L$, we are done. To this end suppose $g \in D_{1}^{d} \cap D$. Then $g \perp h$ for all $h \in D_{1}$, so $g \perp \pi h$ for all $h \in D_{1}$. Since $\pi\left(D_{1}\right)=J$, this implies $g \perp f$ for all $f \in J$, so, in particular, $g \perp \pi^{2} u$ for all $0 \leq u \in D$. Hence, $g \perp \pi^{2} g$ so $\pi^{2} g \perp \pi^{2} g$, 
i.e., $\pi^{2} g=0$. This implies $\pi g \in N_{\pi}$ and, since, $N_{\pi} \subset D_{1}$, it follows from $g \in D_{1}^{d} \subset N_{\pi}^{d}$ that $g \perp \pi g$ and, therefore, $\pi g=0$. Hence $g \in N_{\pi}$ so $g \perp g$, i.e., $g=0$. We have thus shown $D_{1}^{d} \cap D=\{0\}$ and, since $D$ is order dense, this implies $D_{1}$ is order dense. We may conclude, therefore, that $\pi=\pi_{1} \pi^{2}$ in $\operatorname{Orth}^{\infty}(L)$.

Finally, take $\pi \in \operatorname{Orth}^{\infty}(L)$ arbitrary. By the above, there exists $\pi_{1} \in \operatorname{Orth}^{\infty}(L)$ such that $|\pi|=\pi_{1} \pi^{2}$. Now $|\pi|=\left(\pi_{1} \pi\right) \pi$ implies $\pi=$ $\left(\pi_{1} \pi\right)|\pi|$, so $\pi=\left(\pi_{1} \pi\right)\left(\pi_{1} \pi^{2}\right)=\left(\pi_{1}^{2} \pi\right) \pi^{2}$. Hence $\operatorname{Orth}^{\infty}(L)$ is von Neumann regular.

COROllaRY 4.6. If $L$ is a uniformly complete Riesz space, then every weak order unit in $\operatorname{Orth}^{\infty}(L)$ has an inverse in $\operatorname{Orth}^{\infty}(L)$.

Proof. Let $\pi$ be a weak order unit in $\operatorname{Orth}^{\infty}(L)$. By the above theorem there exists $\pi_{1} \in \operatorname{Orth}^{\infty}(L)$ such that $\pi=\pi_{1} \pi^{2}$. Now it follows from $\pi\left(I-\pi_{1} \pi\right)=0$ that $\pi \perp I-\pi_{1} \pi$, hence $I-\pi_{1} \pi=0$, i.e., $\pi_{1}=\pi^{-1}$.

We conclude this paper with a simple characterization of weak order units in $\operatorname{Orth}^{\infty}(L)$.

THEOREM 4.7. If $L$ is an Archimedean Riesz space, then the element $\pi$ in $\operatorname{Orth}^{\infty}(L)$ is a weak order unit iff $\pi$ is injective.

Proof. First assume $0 \leq \pi \in \operatorname{Orth}^{\infty}(L)$ with domain $D$ is injective, and suppose $0 \leq \pi_{0} \in \operatorname{Orth}^{\infty}(L)$ is such that $\pi_{0} \wedge \pi=0$. Then $\pi \pi_{0}=0$, which implies $\pi\left(\pi_{0} f\right)=0$ for all $f \in \pi_{0}^{-1}(D)$. Therefore $\pi_{0} f=0$ for all $f \in \pi_{0}^{-1}(D)$ and, since $\pi_{0}^{-1}(D)$ is order dense, we get $\pi_{0}=0$. Hence $\pi$ is a weak order unit.

Now assume $0 \leq \pi \in \operatorname{Orth}^{\infty}(L)$ with domain $D$ is a weak unit in $\operatorname{Orth}^{\infty}(L)$ and take $0 \leq u \in N_{\pi}$. Put $D_{0}=\{u\}^{d d} \oplus\{u\}^{d}$ and define the mapping $\pi_{0}$ from $D_{0}$ into $L$ by $\pi_{0}(f+g)=f$ for all $f \in\{u\}^{d d}$ and $g \in\{u\}^{d}$. Then $0 \leq \pi_{0} \in \operatorname{Orth}^{\infty}(L)$, and it follows immediately from the definition of $\pi_{0}$ that $\pi \pi_{0} f=0$ for all $f \in D \cap D_{0}$. Therefore $\pi \pi_{0}=0$, which implies $\pi \wedge \pi_{0}=0$ in $\operatorname{Orth}^{\infty}(L)$. Since $\pi$ is a weak order unit, we deduce that $\pi_{0}=0$, so $u=0$. Hence $N_{\pi}=\{0\}$, i.e., $\pi$ is injective.

\section{REFERENCES}

[1] C. D. Aliprantis and O. Burkinshaw, Locally Solid Riesz Spaces, Academic Press, New York-San Francisco-London, 1978.

[2] S. J. Bernau, Orthocompletion of lattice groups, Proc. London Math. Soc., (3) 16 (1966), 107-130. 
[3] _ Lateral and Dedekind completion of Archimedean lattice groups, J. London Math. Soc., 12 (1976), 320-322.

[4] A. Bigard, K. Keimel and S. Wolfenstein, Groupes et Anneaux Réticulés, Lecture Notes in Mathematics 608, Springer-Verlag, Berlin-Heidelberg-New York, 1977.

[5] M. Duhoux and M. Meyer, Extended Orthomorphisms on Archimedean Riesz spaces, Rapport no. 114 (1981), Séminaire de mathémqtique pure, Institut de Mathématique pure et appliquee, Université Catholique de Louvain.

[6] A. W. Hager, Isomorphisms of some completions of $C(X)$, Topology Proc., 4 (1979), 407-435.

[7] C. B. Huijsmans and B. de Pagter, Ideal theory in f-algebras, Trans. Amer. Math. Soc., 269 (1982), 225-245.

[8] J. Lambek, Lectures on Rings and Modules, Blaisdell Publishing Company, Waltham-Toronto-London, 1966.

[9] W. A. J. Luxemburg, Some Aspects of the Theory of Riesz spaces, The University of Arkansas lecture notes in Math., Volume 4, 1979.

[10] W. A. J. Luxemburg and A. R. Schep, A Radon-Nikodym type theorem for positive operators and a dual, Indag. Math., 40 (Proc. Nederl. Acad. Sci. A81), 357-375 (1978).

[11] W. A. J. Luxemburg and A. C. Zaanen, Riesz Spaces I, North-Holland, Amsterdam-London, 1971.

[12] B. de Pagter, f-Algebras and Orthomorphisms, Thesis, Leiden, 1981.

Received March 17, 1982 and in revised form August 27, 1982. Work on this paper was supported by a NATO-Science Fellowship from the Netherlands Organization for the Advancement of Pure Research (Z.W.O.).

California Institute of Technology

PASADENA, CA 91125

Present address: Delf $t$ University of Technology

Delft, The Netherlands 


\section{PACIFIC JOURNAL OF MATHEMATICS \\ EDITORS}

DONALD BABBITT (Managing Editor)

University of California.

Los Angeles, CA 90024

Hugo Rossi

University of Utah

Salt Lake City, UT 84112

C. C. Moore and Arthur Ogus

University of California

Berkeley, CA 94720
J. DugundjI

Department of Mathematics

University of Southern California

Los Angeles, CA 90089-1113

R. FinN and H. Samelson

Stanford University

Stanford, CA 94305

\section{ASSOCIATE EDITORS}
R. ARENS
E. F. BECKENBACH
B. H. NeumanN
F. WOLF
K. YoshidA (1906-1982)

\section{SUPPORTING INSTITUTIONS}

UNIVERSITY OF ARIZONA

UNIVERSITY OF BRITISH COLUMBIA

CALIFORNIA INSTITUTE OF TECHNOLOGY

UNIVERSITY OF CALIFORNIA

MONTANA STATE UNIVERSITY

UNIVERSITY OF NEVADA. RENO

NEW MEXICO STATE UNIVERSITY

OREGON STATE UNIVERSITY
UNIVERSITY OF OREGON

UNIVERSITY OF SOUTHERN CALIFORNIA

STANFORD UNIVERSITY

UNIVERSITY OF HAWAII

UNIVERSITY OF TOKYO

UNIVERSITY OF UTAH

WASHINGTON STATE UNIVERSITY

UNIVERSITY OF WASHINGTON 


\section{Pacific Journal of Mathematics}

Vol. 112, No. $1 \quad$ January, 1984

Richard Blaine Barrar and Henry Loeb, Characterizing the divided difference weights for extended complete Tchebycheff systems $\ldots \ldots \ldots \ldots 1$

Harold Bennett and David John Lutzer, Generalized ordered spaces with

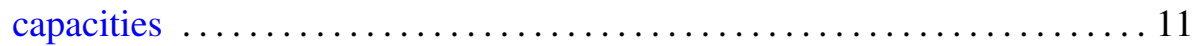

Geoffrey J. Butler and Lynn Harry Erbe, Comparison theorems for second-order operator-valued linear differential equations

Bohumil Cenkl and Richard D. Porter, de Rham theorem with cubical

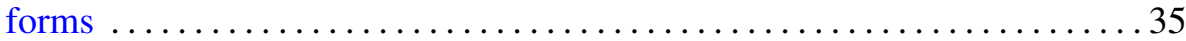

Zvonko Cerin, Characterizing global properties in inverse limits . ....... 49

Thomas Eugene Duchamp and Morris Kalka, Holomorphic foliations and deformations of the Hopf foliation .........................69 69

John Paul Hempel, Homology of coverings $\ldots \ldots \ldots \ldots \ldots \ldots \ldots \ldots \ldots$

Gerald Norman Hile and R. Z. Yeh, Inequalities for eigenvalues of the

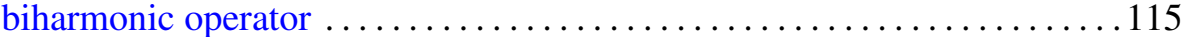

Kenneth Irwin Joy, A description of the topology on the dual space of a

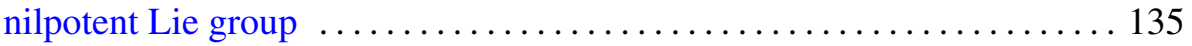

Alex Kumjian, On localizations and simple $C^{*}$-algebras $\ldots \ldots \ldots \ldots \ldots 141$

Bernardus de Pagter, The space of extended orthomorphisms in a Riesz

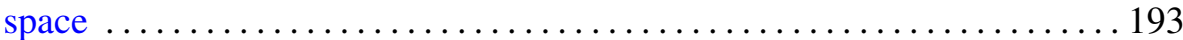

Stephen C. Persek, Iterated averaging for periodic systems with hidden

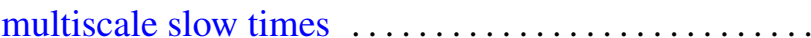

David Francis Rearick, Divisibility of arithmetic functions . . . . . . . . . 237

Masaaki Suzuki, The intrinsic metrics on the circular domains in $\mathbf{C}^{n}$ 